\title{
Immediate Near-Total Scalp Reconstruction with Artificial Dermis on Exposed Calvarium
}

\author{
Sooyeon Park, \\ Ki Taik Han, \\ Min Cheol Kim, \\ Jin Soo Lim \\ Department of Plastic and Reconstructive \\ Surgery, St. Vincent's Hospital, College of \\ Medicine, The Catholic University of Korea, \\ Suwon, Korea \\ No potential conflict of interest relevant to \\ this article was reported.
}

\begin{abstract}
Scalp defect management is complicated secondary to reduced laxity in the scalp and forehead area. For reconstruction of larger defects with exposed bone and loss of the periosteal layer, free flap reconstruction is one option for single-stage surgery, although the procedure is lengthy and includes the possibility of flap loss. We successfully performed a single-stage reconstruction of a large scalp defect using a combination of artificial dermis, split-thickness skin graft, and full-thickness skin graft following wide excision of a cutaneous angiosarcoma, and present our method as one option for the treatment of large oncologic surgical defects in patients who are poor candidates for free flap surgery.
\end{abstract}

Keywords: Scalp reconstruction / Angiosarcoma / Split thickness skin graft / Artificial dermis

\section{INTRODUCTION}

Reconstruction of the scalp and forehead area is clinically complicated owing to the immobility of the skin and galea. For coverage of large defects, split-thickness skin graft (STSG) is the most costeffective and reliable method, although it requires a vascularized wound bed.Subsequently, to perform an STSG over the calvarium, outer table removal is required, which can increase surgical complexity and risk.

We will describe a successful experience with single-stage reconstruction of a large defect using artificial dermis, STSG, and full-thickness skin graft (FTSG) after wide excision of a cutaneous angiosarcoma in the scalp and forehead area.

\section{CASE REPORT}

A 78-year-old woman presented with a superficial red lesion throughout the left forehead, scalp, and periorbital area (Fig. 1).

Correspondence: Jin Soo Lim

Department of Plastic and Reconstructive Surgery, St. Vincent's Hospital, College of Medicine, The Catholic University of Korea, 93 Jungbu-daero, Paldal-gu, Suwon 16247, Korea

E-mail: prsdrlim@yahoo.com

Received April 6, 2016 / Revised December 5, 2016 / Accepted December 5, 2016
She was diagnosed with angiosarcoma on biopsy. No distant metastases were identified on chest computed tomography (CT), brain magnetic resonance imaging, or positron emission tomography (PET)-CT.

Under general anesthesia, wide surgical excision was performed, including the pericranium, resulting in a near total defect of $25 \times 20 \mathrm{~cm}$ (Fig. 2). First, a FTSG was applied over the periorbital area and lower forehead area. Then, micro-burring of the cortical bone was performed followed by the application of Matriderm (Medskin Solution Dr Suwelack AG, Billerbeck, Germany) and afterward, STSG coverage was applied to the remaining defect (Fig. 3). The patient was discharged from the hospital in a tumorfree state 2 weeks postoperatively with no wound complications (Fig. 4). She received radiotherapy and was seen for follow-up in the outpatient department 13 months postoperatively, at which time she reported no other complications. Much to our regret, we lost her so that we could not follow upher wound after that time.

\section{DISCUSSION}

Surgical coverage of a large scalp defect by local flap is challenging because of the inelasticity of scalp and forehead tissue [1,2]. Several 

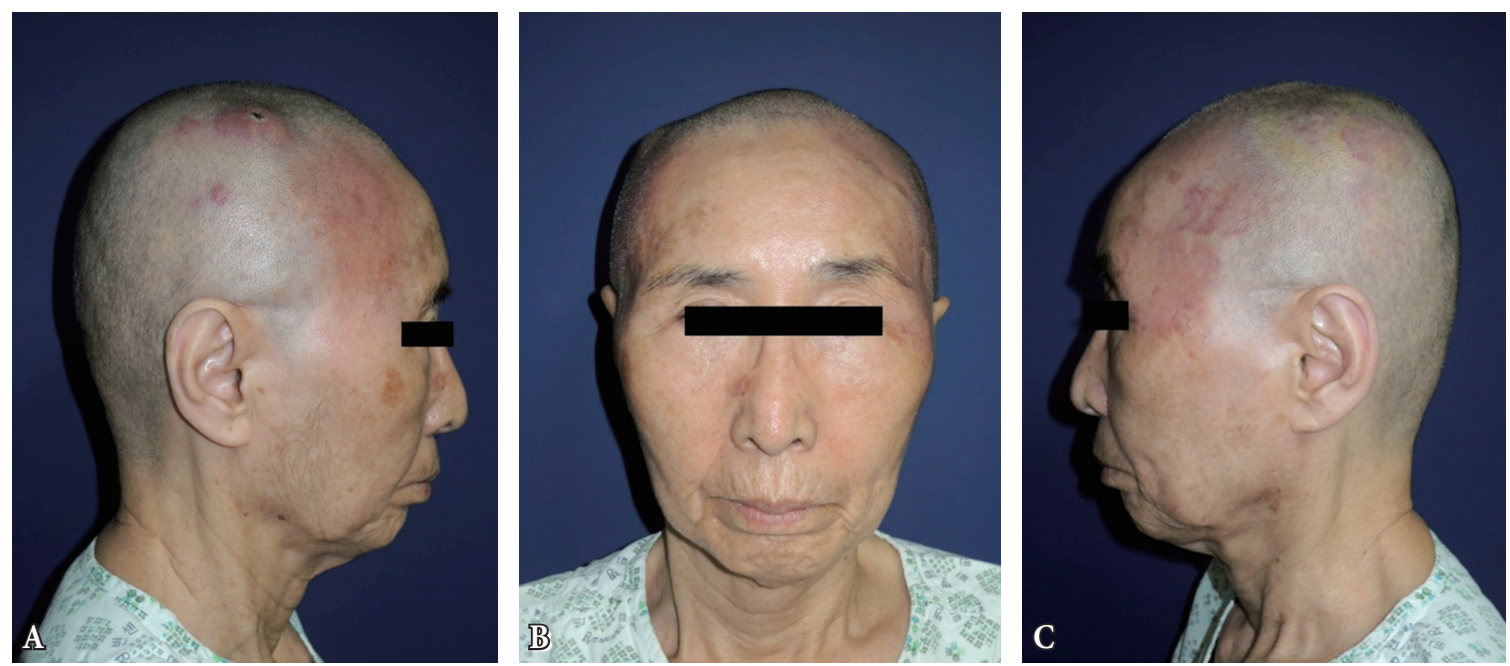

Fig. 1. Preoperative photo: patient with superficial red lesions throughout the left forehead, scalp, and periorbital area. (A) Right side view. (B) Front view. (C) Left side view.
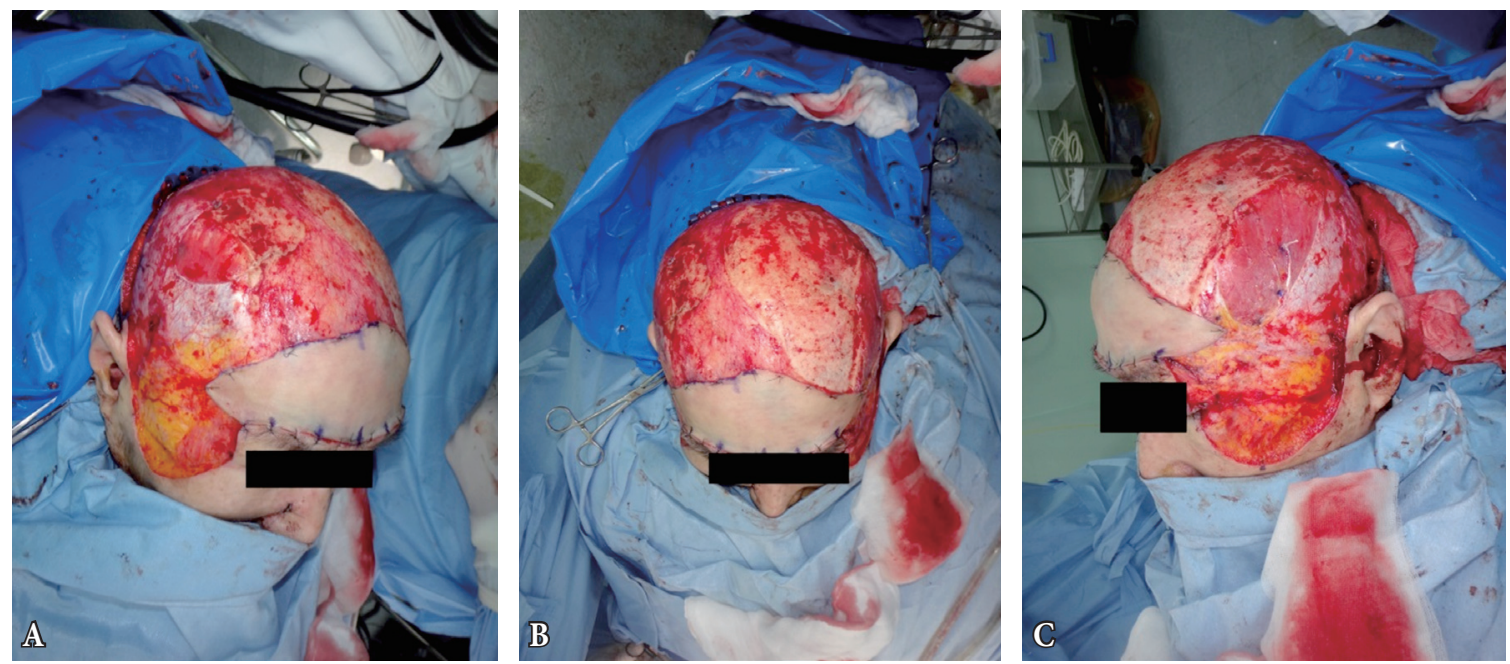

Fig. 2. Surgical photograph: wide surgical excision of the tumor resulting in a near-total scalp defect of $25 \times 20 \mathrm{~cm}$. (A) Right side view. (B) Front view. (C) Left side view.

types of free flaps have been used for scalp reconstruction, such as latissimus dorsi (LD), anterolateral thigh (ALT), radial forearm, rectus abdominis, or omental flap. Overall, LD or ALT flaps are preferred secondary to the large surface area and long vascular pedicle $[3,4]$. However, free tissue transfers require microvascular expertise and are associated with the possibility of postoperative muscle atrophy and contracture as well as the attend and risk of donor site morbidity or abnormality $[2,4]$.

Alternatively, FTSG is associated with lower risk of contracture, but is not appropriate for large defect coverage [1]. STSG is effective for large defect coverage due to minimal donor site morbidity, but again requires a well-vascularized wound bed, and cannot be applied over bone without intact periosteum $[5,6]$. Therefore, twostage reconstruction is needed. The first step involves burring the bone until there is sufficient bleeding for granulation, followed by subsequent STSG. Ultimately, STSG over bare bone requires vascularized neodermis. In these cases, artificial dermis is the preferred grafting tool [6]. 

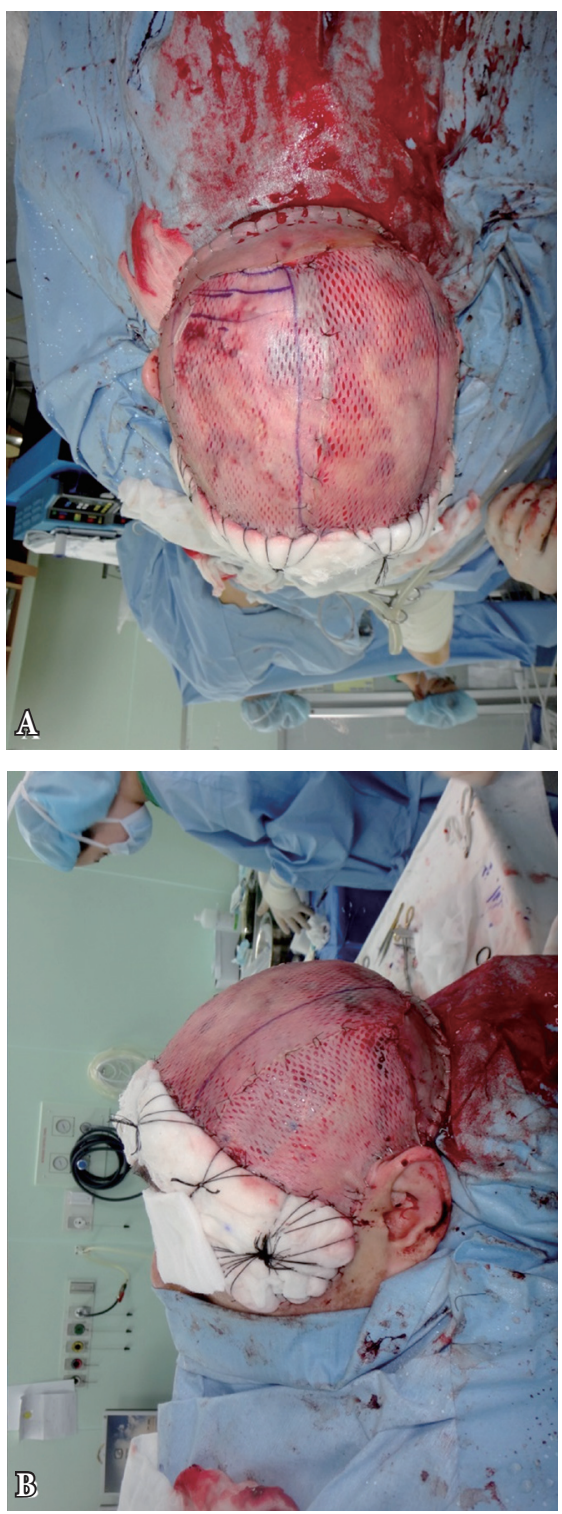

Fig. 3. Surgical photo: cortical burring followed by application of artificial dermis and meshed STSG to cover the defect (the frontal area and occipital area was covered by application of FTSG). (A) Above view. (B) Left side view. STSG, split-thickness skin graft; FTSG, full-thickness skin graft.

Biosynthetic composites have been used in complicated wounds such as burns, and one of the popular dermal substitutes is acellular dermal matrix. Matriderm is a highly porous membrane composed of three-dimensional-coupled collagen and elastin. The collagen matrix is derived from bovine dermis, and the elastinis derived from the hydrolysis of bovine nuchal ligament.
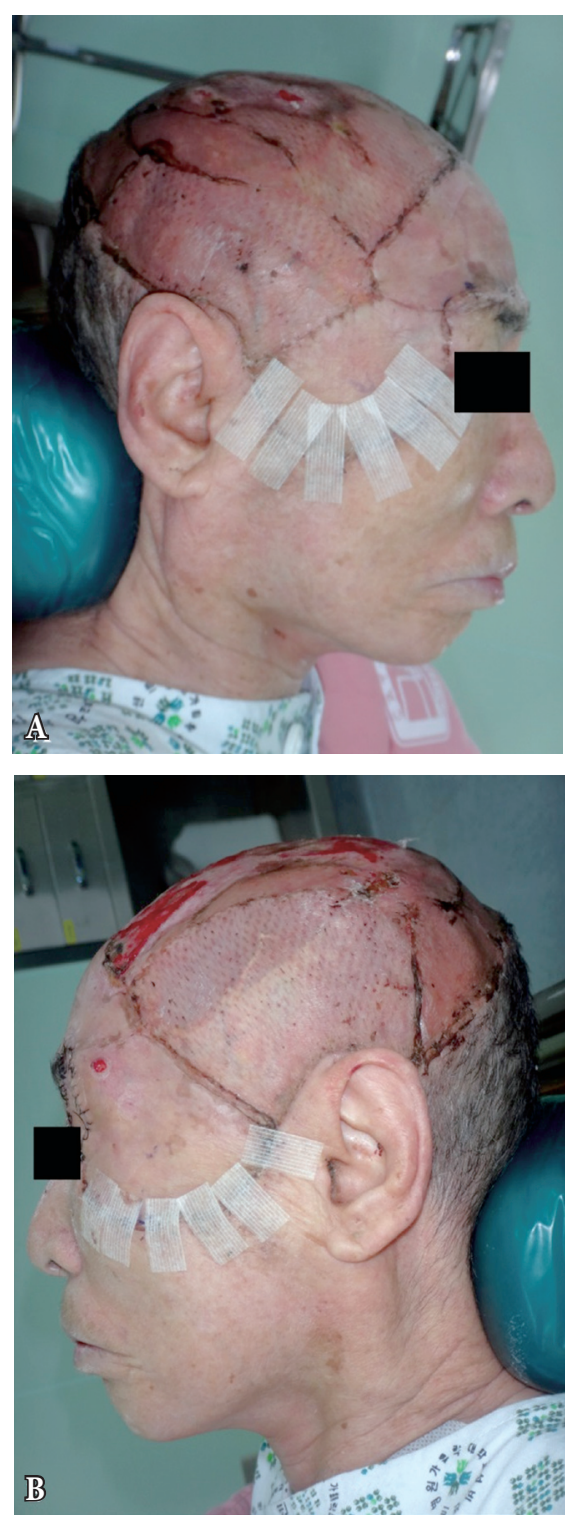

Fig. 4. Postoperative photo: the patient was discharged 2 weeks postoperatively in a tumor-free state without complications. (A) Right side view. (B) Left side view.

Matriderm acts as a platform for skin regeneration, assisting the dermal wound bed and supporting the development of rete ridges and capillary loops in the regenerated skin [6-9].

Over bony prominences and calvarium with an intact periosteal layer, STSG can be unstable, resulting in breakdown in these areas. Oncologic surgical defects require stable coverage because 
of the possibility of ulceration following radiation therapy [1,5]. In those cases, the use of artificial dermis provides stability and can reduce ulcer formation.

Angiosarcoma is a rare vascular tumor involving the endothelial cells, and cutaneous angiosarcoma is known to occur in the face and scalp area. This tumor has highly aggressive malignant characteristics including early metastasis and local regional recurrence. Standard treatment involves wide excision and radiation therapy, and typically, the periosteum is stripped to reduce local recurrence [10].

For patients with extensive angiosarcomas, wide excision and primary reconstruction alone is a burden. Furthermore, tumor margins that appear free in frozen section biopsy during surgery may in fact be revealed to be close or positive on definitive permanent section analysis, in which case, the patient will possibly require a second operation and further resection.

We propose that one-step reconstruction of an oncologic surgical scalp defect with artificial dermis and skin grafting is an excellent option over free tissue surgery or a two-stage skin graft for older patients with co-morbidities.

\section{REFERENCES}

1. Tufaro AP, Buck DW 2nd, Fischer AC. The use of artificial dermis in the reconstruction of oncologic surgical defects. Plast Reconstr Surg 2007;120:638-46.
2. Cho JY, Jang YC, Hur GY, Koh JH, Seo DK, Lee JW, et al. One stage reconstruction of skull exposed by burn injury using a tissue expansion technique. Arch Plast Surg 2012;39:118-23.

3. Eck DL, Koonce SL, Al Majed BM, Perdikis G. Evaluation of options for large scalp defect reconstruction: a 12-year experience. Eplasty 2014;14:e10.

4. Sosin M, De la Cruz C, Bojovic B, Christy MR, Rodriguez ED. Microsurgical Reconstruction of Complex Scalp Defects: An Appraisal of Flap Selection and the Timing of Complications. J Craniofac Surg 2015;26:1186-91.

5. Ioannides C, Fossion E, McGrouther AD. Reconstruction for large defects of the scalp and cranium. J Craniomaxillofac Surg 1999;27:145-52.

6. Bertolli E, Campagnari M, Molina AS, Macedo MP, Pinto CA, Cunha IW, et al. Artificial dermis (Matriderm(R)) followed by skin graft as an option in dermatofibrosarcoma protuberans with complete circumferential and peripheral deep margin assessment. Int Wound J 2015;12:545-7.

7. Min JH, Yun IS, Lew DH, Roh TS, Lee WJ. The use of matriderm and autologous skin graft in the treatment of full thickness skin defects. Arch Plast Surg 2014;41:330-6.

8. Hamuy R, Kinoshita N, Yoshimoto H, Hayashida K, Houbara S, Nakashima M, et al. One-stage, simultaneous skin grafting with artificial dermis and basic fibroblast growth factor successfully improves elasticity with maturation of scar formation. Wound Repair Regen 2013;21:141-54

9. Jeon H, Kim J, Yeo H, Jeong H, Son D, Han K. Treatment of diabetic foot ulcer using matriderm in comparison with a skin graft. Arch Plast Surg 2013;40:403-8.

10. Choi JH, Ahn KC, Chang H, Minn KW, Jin US, Kim BJ. Surgical treatment and prognosis of angiosarcoma of the scalp: a retrospective analysis of 14 patients in a single institution. Biomed Res Int 2015;2015:321896. 